Monolithic scintillator PET detectors with intrinsic depth-of-interaction correction

This article has been downloaded from IOPscience. Please scroll down to see the full text article.

2009 Phys. Med. Biol. 541893

(http://iopscience.iop.org/0031-9155/54/7/003)

View the table of contents for this issue, or go to the journal homepage for more

Download details:

IP Address: 131.180.130.114

The article was downloaded on 21/12/2010 at 10:42

Please note that terms and conditions apply. 


\title{
Monolithic scintillator PET detectors with intrinsic depth-of-interaction correction
}

\author{
Marnix C Maas ${ }^{1}$, Dennis R Schaart ${ }^{1}$, D J (Jan) van der Laan ${ }^{1}$, \\ Peter Bruyndonckx ${ }^{2}$, Cedric Lemaître ${ }^{2}$, Freek $\mathbf{J}$ Beekman $^{1,3}$ and \\ Carel W E van Eijk ${ }^{1}$ \\ ${ }^{1}$ Delft University of Technology, Mekelweg 15, 2629 JB Delft, The Netherlands \\ 2 Vrije Universiteit Brussel, Pleinlaan 2, 1050 Brussels, Belgium \\ ${ }^{3}$ University Medical Centre Utrecht, Heidelberglaan 100, Utrecht, The Netherlands \\ E-mail: d.r.schaart@tudelft.nl
}

Received 12 October 2008, in final form 6 February 2009

Published 5 March 2009

Online at stacks.iop.org/PMB/54/1893

\begin{abstract}
We developed positron emission tomography (PET) detectors based on monolithic scintillation crystals and position-sensitive light sensors. Intrinsic depth-of-interaction (DOI) correction is achieved by deriving the entry points of annihilation photons on the front surface of the crystal from the light sensor signals. Here we characterize the next generation of these detectors, consisting of a $20 \mathrm{~mm}$ thick rectangular or trapezoidal LYSO:Ce crystal read out on the front and the back (double-sided readout, DSR) by Hamamatsu S8550SPL avalanche photodiode (APD) arrays optimized for DSR. The full width at half maximum (FWHM) of the detector point-spread function (PSF) obtained with a rectangular crystal at normal incidence equals $\sim 1.05 \mathrm{~mm}$ at the detector centre, after correction for the $\sim 0.9 \mathrm{~mm}$ diameter test beam of annihilation photons. Resolution losses of several tenths of a mm occur near the crystal edges. Furthermore, trapezoidal crystals perform almost equally well as rectangular ones, while improving system sensitivity. Due to the highly accurate DOI correction of all detectors, the spatial resolution remains essentially constant for angles of incidence of up to at least $30^{\circ}$. Energy resolutions of $\sim 11 \%$ FWHM are measured, with a fraction of events of up to $75 \%$ in the full-energy peak. The coincidence timing resolution is estimated to be $2.8 \mathrm{~ns}$ FWHM. The good spatial, energy and timing resolutions, together with the excellent DOI correction and high detection efficiency of our detectors, are expected to facilitate high and uniform PET system resolution.
\end{abstract}

(Some figures in this article are in colour only in the electronic version) 


\section{Introduction}

To address the need for improved image quality in preclinical and clinical molecular imaging, various new scintillation detectors for positron emission tomography (PET) have recently been proposed. Many PET applications benefit from enhanced system resolution, and much effort has therefore been spent on improving detector spatial resolution.

An important cause for resolution loss is the varying depth of interaction (DOI) of annihilation quanta within the scintillation crystals. The resulting parallax errors cause a nonuniformly degraded system resolution. Therefore, it is desirable that a PET detector offers some form of DOI correction. In addition, good energy and timing resolutions as well as high detection efficiency are crucial to obtain high image quality. The relative importance of each of these performance parameters depends on the specific application and subject size. In small-animal systems for example, image quality is very much determined by the detector spatial resolution and DOI effects. In clinical applications, image quality suffers from intrapatient scatter and random coincidences, the effects of which can be reduced if the detectors have good energy and timing resolutions.

Many high-resolution PET detector designs rely on relatively long $(\sim 1 \mathrm{~cm})$, thin ( $\sim 1 \mathrm{~mm}$ ) scintillation crystals (Lecomte et al 1996, Bloomfield et al 1997, Del Guerra et al 1998, Correia et al 1999, Weber et al 1999, Surti et al 2003, Tai et al 2003, 2005). Although such designs aim to improve resolution by reducing or eliminating light spreading, their performance is still limited by inter-crystal scattering. Furthermore, the reflective material between the crystals increases the fraction of dead space. Finally, without DOI determination, one needs to compromise between high system resolution (shorter crystals to prevent excessive DOI errors) and sensitivity (longer crystals to improve the detection efficiency).

Other groups have therefore developed (non-monolithic) detectors with DOI determination. The approaches taken include estimating the DOI from the ratio of the signals measured at opposite sides of the crystal (Wang et al 2004, Abreu et al 2006, Du et al 2008), phoswhich approaches (Seidel et al 2003, Ziemons et al 2005), multi-layer pixelated scintillators with optical DOI encoding (Orita et al 2005) and radial stacks of individual detector modules (McElroy et al 2005). In these designs, the benefit of DOI information is often gained at the expense of other performance parameters. Light losses due to the use of small (pixelated) crystals result in energy resolutions $>20 \%$ in most and $>40 \%$ in some designs. In addition, the dead space between detector modules and crystal elements decreases the system sensitivity. Furthermore, the manufacturing costs of designs based on many small crystals tend to increase with decreasing crystal cross-section.

Detectors with monolithic scintillators coupled to one or more position-sensitive light sensors can offer a solution to the above issues (Clément et al 1998, Bruyndonckx et al 2003). In these detectors, the entry point of an annihilation photon on the front surface of the crystal can be derived from the measured light distribution, for example using a statistical nearestneighbour algorithm. By deriving the entry point, the depth of interaction is intrinsically corrected for. At the same time, detection efficiency is maximized as there is no dead space within a monolithic crystal. Moreover, when compact, solid-state light sensors such as avalanche photodiode (APD) arrays are used instead of bulky photomultiplier tubes (PMTs), the detectors can be placed closely together to optimize scanner sensitivity. As an added advantage, the use of solid-state light sensors opens up the possibility of integrating the PET system with a magnetic resonance imaging (MRI) device (e.g. Catana et al 2006, Judenhofer et al 2008, Woody et al 2007).

Preliminary results obtained with early monolithic scintillator detector prototypes have been reported previously (Maas et al 2006). These indicated that $10 \mathrm{~mm}$ thick monolithic 


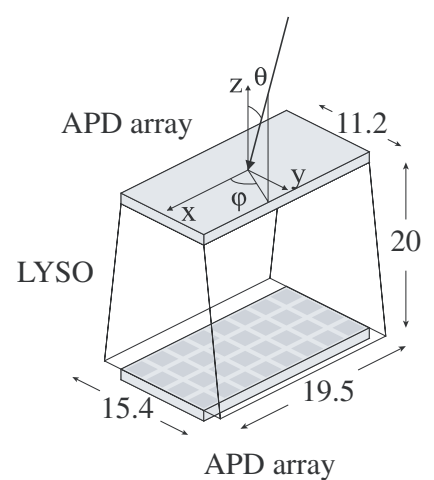

(a)

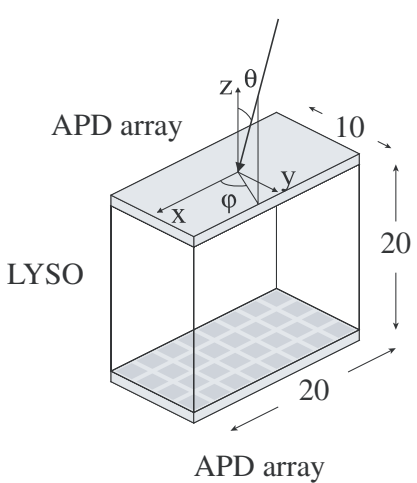

(b)

Figure 1. Schematic representation of the trapezoidal (a) and rectangular (b) detector geometries with double-sided APD array readout. In both drawings, the arrow indicates the path of an annihilation photon incident on the front surface of the detector. The coordinate system used to specify the entry point and angle of incidence is also indicated. Dimensions are in $\mathrm{mm}$.

LYSO:Ce crystals perform better with the light sensor placed on the front surface (frontside readout, FSR) than with conventional backside readout (BSR), since the majority of the detected annihilation photons interact in the front half of the crystal. The same study indicated that $20 \mathrm{~mm}$ thick monolithic crystals read out on both the front and back surfaces (doublesided readout, DSR) may offer similarly good spatial resolution as $10 \mathrm{~mm}$ thick crystals with FSR, with the added benefit of significantly increased detection efficiency. It is emphasized that unconventional readout geometries such as FSR and DSR are made possible by the fact that solid-state light sensors such as APD arrays are essentially transparent to $511 \mathrm{keV}$ photons.

The present study aims to further explore the benefits of double-sided readout. We fully characterize the performance of the next generation of monolithic detectors, read out by two Hamamatsu S8550SPL APD arrays in DSR geometry. This APD array, based on the commonly available S8550 array, is a customized product optimized specifically for FSR and DSR (Abreu et al 2006). The performance obtained with $20 \mathrm{~mm}$ thick rectangular and trapezoidal LYSO:Ce crystals is compared. Especially in small-bore, high-resolution systems, trapezoidal crystals significantly improve system sensitivity by minimizing the dead space within a detector ring (van der Laan et al 2007).

For the first time, the two-dimensional (2D) detector point-spread function (PSF) of our detectors is reported. We furthermore use a new method to correct the measured PSF for the influence of the finite test beam diameter, in order to estimate the detector PSF applicable to individual annihilation photons. In addition, the intrinsic DOI correction of the detectors is studied in detail by varying the angle of incidence in different directions. Finally, the energy and timing resolutions of the detectors are reported.

\section{Materials and methods}

\subsection{Detector hardware}

Each detector consists of a $20 \mathrm{~mm}$ thick monolithic LYSO:Ce crystal (Crystal Photonics) and two position-sensitive Hamamatsu S8550SPL APD arrays in DSR geometry, as indicated in figure 1. The crystal dimensions are indicated in the same figure. The APD arrays are optically 
coupled to the front and back crystal surfaces using Meltmount (Cargille Laboratories, Cedar Grove, NJ, USA). The side surfaces are wrapped in highly reflective Teflon tape.

The performance of trapezoidal crystals (see figure 1(a)), designed for a ring of 32 detectors with an inner diameter of $123.8 \mathrm{~mm}$, is compared to a rectangular crystal (figure 1(b)) that was selected for its good performance in preliminary studies. Trapezoidal crystals with polished and unpolished (as-cut) surfaces are compared. Crystals are identified by a code consisting of, respectively, a character indicating the crystal geometry (' $R$ ' for rectangular, ' $\mathrm{T}$ ' for trapezoidal), a sample number, and either the character ' $\mathrm{P}$ ' for optically polished crystals or ' $A$ ' for crystals that received no surface treatment after cutting.

The Hamamatsu S8550SPL is a customized version of the commonly available S8550 array. Both are $4 \times 8$ arrays of $1.6 \times 1.6 \mathrm{~mm}^{2}$ pixels at a pitch of $2.3 \mathrm{~mm}$, subdivided into two banks of $2 \times 8$ pixels with a common cathode per bank. In the S8550SPL, the length of the metal readout pins on the back of the device has been reduced from $4.57 \mathrm{~mm}$ to $2.60 \mathrm{~mm}$, minimizing the amount of material between radiation source and crystal if the array is placed on the crystal front surface, as in FSR and DSR geometries (Abreu et al 2006). Furthermore, the protective epoxy resin coating on the photosensitive surface is thinner $(0.45 \mathrm{~mm}$ instead of $0.60 \mathrm{~mm}$ ) and has been polished to a flatness of $\pm 10 \mu \mathrm{m}$ to facilitate good optical coupling to the crystal. Finally, the dark currents of the arrays used here (serial numbers 036 and 037 ) are an estimated factor of 2 lower than those of the $\$ 8550$ arrays used in our previous experiments (Maas et al 2006), resulting in better detector performance (Maas et al 2008).

For each of the two pixel banks within an array, the manufacturer specifies the bias voltage $V_{50}$ (typically $\sim 400 \mathrm{~V}$ ) at which the APD gain $M=50$. Differences in $V_{50}$ of up to $\sim 20 \mathrm{~V}$ are common between banks within the same array and between different arrays. We therefore express the bias voltage per bank in terms of

$$
\Delta V \equiv V-V_{50}
$$

where $V$ is the applied bias voltage. Figure 2 shows the average APD gains per bank of the APD arrays used, measured as a function of $\Delta V$ with $5.89 \mathrm{keV}$ x-rays from a ${ }^{55} \mathrm{Fe}$ source. If all banks are biased at the same value of $\Delta V$, their average gains coincide within $\sim 4 \%$ over the range of bias voltages considered. Furthermore, gain variations on the order of $\sim 10 \%$ are commonly observed between individual APD pixels within one bank. It has been confirmed in preliminary experiments that this does not influence the detector spatial resolution, as the position estimation (section 2.3) is based on reference data measured at the same gain distribution. The effect on the detector energy resolution also appeared to be negligible $(<0.1 \%$ FWHM). No corrections for gain non-uniformities have therefore been applied in this work.

\subsection{Intrinsic DOI correction}

In the absence of scattering, the path of a gamma-photon can be described by the coordinates at which it intersects the front surface of the detector (the 'entry point') and its angle of incidence, see figure 1. In the present detector concept, the entry point of each detected annihilation photon (event) is estimated from the distribution of the scintillation light measured by the position-sensitive light sensors. This is done by means of a statistical algorithm that uses sets of reference data containing the measured light distributions of a large number of events recorded at known entry points, with each reference set corresponding to a particular angle of incidence. In a PET system, the angle of incidence can be derived from the positions of the two detectors triggering in coincidence (Bruyndonckx et al 2004). Estimating the entry point rather than the point of interaction within the crystal has the advantage of eliminating parallax errors, even though the depth of interaction is not explicitly determined. 




Figure 2. Average APD gains of each $2 \times 8$ pixel bank of the Hamamatsu S8550SPL arrays (serial numbers 036 and 037) as a function of $\Delta V$ (see equation (1)), measured with $5.89 \mathrm{keV}$ x-ray photons directly incident on the APDs.

\subsection{Position estimation}

Reference data are collected by irradiating the detector with $511 \mathrm{keV}$ photons at a series of known positions $\vec{x}_{i}=\left(x_{i}, y_{i}\right)$, see figure 1 , and recording the light distributions of $n_{\text {ref }}$ reference events at each position. This is repeated for various angles of incidence. The light distribution of each event in the reference set is subsequently normalized such that the sum of all detector signals equals unity.

The entry point of an unknown annihilation photon can now be estimated by calculating the sum-of-squares difference of its normalized light distribution with those of all events in the reference set recorded at the appropriate angle of incidence. A subset of the reference data consisting of the $L$ closest matches ('nearest neighbours') is selected, and the most frequently occurring entry point within this subset is assigned to the unknown event. This method is known as the $L$-nearest neighbour method. It has the advantage that the probability of misclassification approaches the theoretical minimum, the Bayes error probability, for sufficiently large reference sets (Cover and Hart 1967). The algorithm can therefore be expected to yield results close to the best achievable with the available reference data, provided that a suitable value of $L$ be used. A method for finding this value has been described elsewhere (Maas et al 2006).

\subsection{Measurement setup}

The measurement setup is shown schematically in figure 3. The detectors are contained in a light-tight, temperature-controlled aluminium box. The temperature $T$ is measured with an LM35D temperature sensor close to one of the APD arrays. A test beam of $511 \mathrm{keV}$ photons is defined by a $0.5 \mathrm{~mm}$ diameter ${ }^{22} \mathrm{Na}$ source and a second detector in coincidence with the APD detector, consisting of a $35 \mathrm{~mm}$ thick BGO crystal (diameter $19 \mathrm{~mm}$ ) coupled to an XP2020 PMT equipped with a $60 \mathrm{~mm}$ thick $\mathrm{Pb}$ collimator with a $5 \mathrm{~mm}$ diameter aperture. The diameter $d_{\mathrm{b}}$ of the test beam, defined as the full width at half maximum (FWHM) of the annihilation 


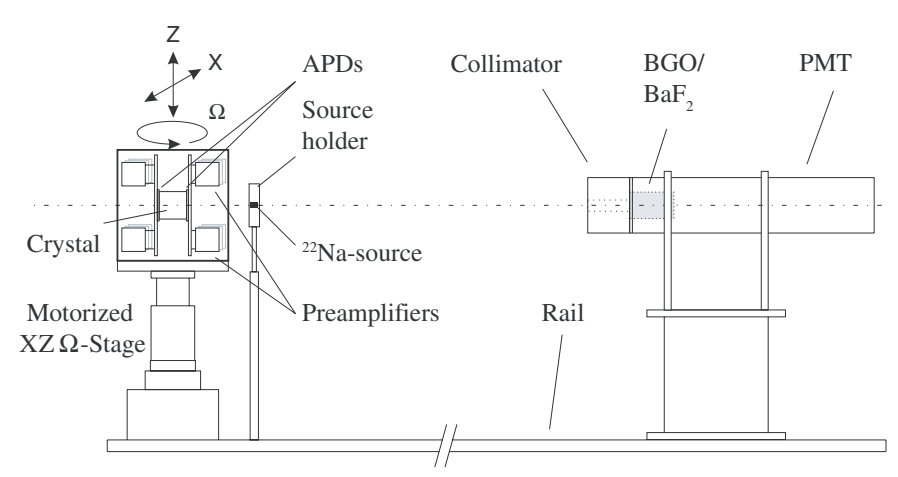

Figure 3. Schematic representation of the setup used for the experimental characterization of the detectors.

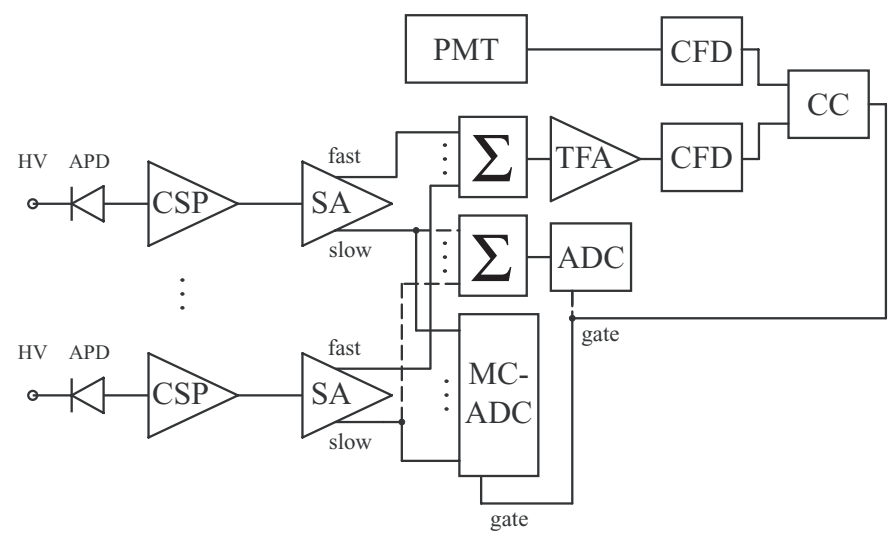

Figure 4. Schematic representation of the readout electronics. Components are denoted by CSP: charge-sensitive preamplifier; SA: shaping amplifier; MC-ADC: multichannel ADC; $\Sigma$ : summing amplifier; TFA: timing filter amplifier; CFD: constant-fraction discriminator; CC: coincidence circuit.

photon fluence profile at the front surface of the crystal (typical value $\sim 1 \mathrm{~mm}$ ), can be varied by varying the distances between source, PMT and APD detector. A computer-controlled $\mathrm{XZ} \Omega$-stage enables irradiating the detector at different positions and angles of incidence.

The readout electronics are schematically shown in figure 4. Each of the 64 APD signals is pre-amplified by a Cremat CR-110 charge-sensitive preamplifier (CSP). Further amplification and shaping is provided by four CAEN N568BB 16-channel shaping amplifiers (SAs), offering a fast and a slow output for each channel. The fast outputs are fixed-gain, single-differentiation stages with a time constant of $100 \mathrm{~ns}$; the slow outputs offer adjustable gain and shaping times $\tau=0.1,0.2,1$ and $3 \mu$ s. Multichannel peak-sensing analogue-to-digital converters (ADCs) (CAEN V785) are used to individually read out the slow outputs. Triggering is performed on the analogue sum signal of the fast outputs, obtained with a summing amplifier.

\subsection{Detector characterization}

2.5.1. Scintillator light yield. The absolute light yields of the LYSO:Ce crystals were measured on a calibrated PMT setup, using a shaping time of $1 \mu \mathrm{s}$. The crystals, wrapped in 
Teflon tape, were irradiated with $662 \mathrm{keV}$ photons from a ${ }^{137} \mathrm{Cs}$ source, and the photoelectron yield was obtained by comparison of the position of the full-energy peak with the singleelectron spectrum. Absolute light yields were calculated by correcting for the effective PMT reflectivity and quantum efficiency (de Haas et al 2005).

2.5.2. Detector energy resolution. The energy resolutions of the assembled detectors were determined by irradiating them with a $\sim 5 \mathrm{~mm}$ diameter beam of $511 \mathrm{keV}$ photons aimed at the detector centre. Pulse height spectra were measured by connecting the CAEN N568BB slow outputs via a summing amplifier to an Ortec AD413 A peak-sensing ADC, see figure 4. FWHM energy resolutions were obtained by fitting the full-energy peaks with Gaussians. All energy resolution measurements were performed at $\Delta V=0 \mathrm{~V}, \tau=0.2 \mu \mathrm{s}$ and at temperatures between $T=21.6^{\circ} \mathrm{C}$ and $T=25.2^{\circ} \mathrm{C}$ with temperature variations of less than $\pm 0.2{ }^{\circ} \mathrm{C}$ during each measurement.

2.5.3. Timing resolution. The detector timing resolution was measured against a $25.4 \mathrm{~mm}$ thick $\mathrm{BaF}_{2}$ crystal (diameter $25.4 \mathrm{~mm}$ ) coupled to an XP2020Q PMT. The PMT signal was directly fed into an Ortec 934 constant fraction discriminator (CFD). Time pickoff on the APD detector was performed by connecting the CAEN N568BB fast outputs via a summing amplifier and an Ortec 454 timing filter amplifier (TFA) with adjustable differentiation and integration time constants to an Ortec 934 CFD, see figure 4. An integration time constant of 2 ns was used, while the differentiation time constant was set to 'out'. The timing spectrum was recorded using an Ortec 476 time-to-amplitude converter (TAC) and an Ortec AD413A peaksensing ADC. The time axis was calibrated with an Ortec 462 time calibrator. The FWHM timing resolution was obtained by fitting the spectrum with a Gaussian. The measurement was performed at $\Delta V=19 \mathrm{~V}, E_{\mathrm{th}} \approx 250 \mathrm{keV}$ and $T=24.3 \pm 0.2{ }^{\circ} \mathrm{C}$.

2.5.4. Spatial resolution measurements. To determine the detector PSF, reference data were first collected by irradiating the crystal front surface at a rectangular grid of equidistant beam positions $\vec{x}_{i}=\left(x_{i}, y_{i}\right)$ and recording a number of $n_{\text {ref }}$ events at each beam position (the coordinate system is indicated in figure 1). The PSF in a test position $\vec{x}_{\mathrm{t}}$ chosen from the positions $\vec{x}_{i}$ was then estimated as follows. An event $j$ recorded at $\vec{x}_{\mathrm{t}}$ was selected as a test event and its position was estimated with the nearest-neighbour procedure described in section 2.3, using the remaining $n_{\text {ref }}-1$ events recorded at $\vec{x}_{t}$, as well as all events collected at all other positions $\vec{x}_{i} \neq \vec{x}_{\mathrm{t}}$, as reference data. This procedure was repeated for all of the $n_{\text {ref }}$ events recorded at $\vec{x}_{\mathrm{t}}$ (leave-one-out approach). The normalized, two-dimensional (2D) histogram of the errors $\Delta \vec{x}_{j}=\hat{\vec{x}}_{j}-\vec{x}_{\mathrm{t}}$, where $\hat{\vec{x}}_{j}$ is the estimated position of the $j$ th event recorded at $\vec{x}_{\mathrm{t}}$, then approaches the point-spread function $\operatorname{PSF}(x, y)$ at $\vec{x}_{\mathrm{t}}$ if the number of test events is sufficiently large. As the PSF can be determined at any of the beam positions $\vec{x}_{i}$, this procedure was also used to e.g. study the FWHM of the PSF as a function of entry position and to derive the average FWHM over the detector surface.

It is to be noted that the measured histogram still includes the influence of the test beam diameter $d_{\mathrm{b}}(\sim 1 \mathrm{~mm}$ FWHM, see section 2.4$)$. In the remainder of this work, the measured result will therefore be denoted as the uncorrected point-spread function $\operatorname{PSF}_{\mathrm{b}}(x, y)$, or, briefly, $\mathrm{PSF}_{\mathrm{b}}$, to distinguish it from the corrected result that will be written without the subscript ' $\mathrm{b}$ ' (see section 2.5.5).

We studied the influence of various parameters (such as the crystal type, the angle of incidence, etc) on the spatial resolution, by varying the parameter of interest in a series of measurements. For efficiency, this was done in a one-dimensional (1D) form of the above 
experiment, in which reference data were collected only along one of the crystal axes (see figure 1) and only the coordinate corresponding to that axis was taken into consideration. Specifically, data were collected at e.g. $\vec{x}_{i}=\left(x_{i}, y=0\right)$; see figure 1 for the coordinate system used. The $x$-coordinates of the events recorded at test position $\vec{x}_{\mathrm{t}}=\left(x_{\mathrm{t}}, y=0\right)$ were estimated, and a normalized 1D histogram of the errors $\Delta x_{j}=\hat{x}_{j}-x_{\mathrm{t}}$ was derived. This histogram will be referred to as the one-dimensional point-spread function 1D-PSF $(x)$, where the subscript ' $b$ ' again indicates that the result still includes the influence of the beam

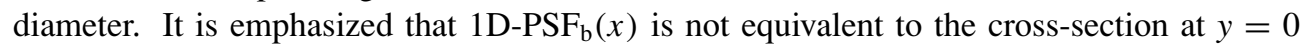
of the two-dimensional $\operatorname{PSF}_{\mathrm{b}}(x, y)$, and that results from $1 \mathrm{D}$ and $2 \mathrm{D}$ measurements are not directly comparable.

All spatial resolution measurements were performed with the setup described in section 2.4. The distance between the beam positions in the $x$ - and/or the $y$-direction (as applicable) was kept at $0.25 \mathrm{~mm}$ in all cases. A number of $n_{\text {ref }}=1500$ events were recorded per beam position in 1D PSF measurements. This value has previously been shown sufficient for good results (Maas et al 2006). In 2D PSF measurements, the symmetry of the crystals was used to increase efficiency: test data were selected from one half $(y \geqslant 0)$ of the crystal only, while reference data were collected for $y \geqslant-2 \mathrm{~mm}$ to avoid truncating the error histograms at $y=0$. At each grid position, $n_{\text {ref }}=500$ events were recorded. For non-perpendicular angles of incidence, the range of beam positions included the crystal front surface as well as the side surface turned towards the beam. The oblique sides of the trapezoidal crystals (see figure 1) were included in all measurements performed in the $y$-direction.

All spatial resolution measurements were performed at $\Delta V=0 \mathrm{~V}, \tau=0.2 \mu \mathrm{s}$ and at temperatures between $T=21.4{ }^{\circ} \mathrm{C}$ and $T=25.3{ }^{\circ} \mathrm{C}$ with temperature variations of less than $\pm 0.2^{\circ} \mathrm{C}$ during each measurement. Furthermore, all events above the relatively low energy threshold $E_{\mathrm{th}} \approx 100 \mathrm{keV}$ were accepted.

2.5.5. Correction for test beam diameter. The relevant quantity for characterizing the spatial resolution of the present type of detector when used in a PET system is the 2D error probability distribution of the entry point assigned to individual annihilation photons, i.e. the detector PSF. To estimate this quantity from the measured distribution $\mathrm{PSF}_{\mathrm{b}}$ described in the previous section, we need to correct for the influence of the test beam diameter $d_{\mathrm{b}}(\sim 1 \mathrm{~mm}$ FWHM, see section 2.4). However, correcting by straightforward deconvolution has appeared to be difficult, amongst others because of divergence of the beam $(\sim 21 \%$ over $20 \mathrm{~mm})$.

Instead, a correction was made using a simple model of the PSF that has been presented and validated elsewhere (Maas 2008). For convenience, we summarize the model briefly in the following. The model is valid in the central part of the detector and describes the PSF as a convolution of two 2D distributions plus a small background. The first distribution, $\gamma$, describes the spatial distribution (projected onto the $x y$ plane) of the energy deposited by annihilation photons within the crystal due to interaction processes such as Compton scattering and x-ray fluorescence. The second distribution, $N_{\Sigma}$, accounts for the positioning error resulting from the finite signal-to-noise ratio of the detector signals, which is determined by statistical variations in the number of photons produced per scintillation event and the fraction of photons detected, by the APD excess noise factor and dark current, by electronic noise, etc (see e.g. Maas et al (2008)).

The 2D distribution $\gamma$ is estimated by means of a Monte Carlo simulation (Maas 2008). This has been done both for the case of a 'pencil' beam with zero diameter, the result of which is denoted by $\gamma_{0}$, and for a detailed model of the test beam used in our experiments (Maas 2008), the result of which is denoted by $\gamma_{\mathrm{b}}$. 
Table 1. Absolute light yield of the crystals investigated, and corresponding energy resolutions measured with a ${ }^{22} \mathrm{Na}$ source $(511 \mathrm{keV})$. The $2 \sigma$-uncertainties are $\sim 10 \%$ for the light yields and $\sim 0.2 \%$ FWHM for the energy resolutions.

\begin{tabular}{lllll}
\hline Crystal & Shape & Surface & $\begin{array}{l}\text { Crystal light yield } \\
(\mathrm{ph} / \mathrm{MeV} \text { at } 662 \mathrm{keV})\end{array}$ & $\begin{array}{l}\text { Detector energy resolution } \\
(\% \text { FWHM at 511 keV })\end{array}$ \\
\hline R01-P & Rectangular & Polished & $20.2 \times 10^{3}$ & 10.5 \\
T02-P & Trapezoid & Polished & $22.8 \times 10^{3}$ & 11.0 \\
T03-A & Trapezoid & As-cut & $21.5 \times 10^{3}$ & 10.5 \\
\hline
\end{tabular}

The distribution $N_{\Sigma}$ is modelled by a $2 \mathrm{D}$ Gaussian function with covariance matrix $\boldsymbol{\Sigma}$, where the non-diagonal elements of $\boldsymbol{\Sigma}$ are assumed to be zero. Results given elsewhere (Maas 2008), as well as the good agreement between model and experiment presented in section 3.3.2, indicate that this is a valid approximation. $\Sigma$ is found by modelling $\operatorname{PSF}_{\mathrm{b}}(x, y)$ as a convolution of $\gamma_{\mathrm{b}}$ and $N_{\Sigma}$, using the diagonal elements of $\Sigma$ as fit parameters. $\operatorname{PSF}(x, y)$ is subsequently estimated as a convolution of $N_{\Sigma}$ and $\gamma_{0}$.

\section{Results and discussion}

\subsection{Energy resolution}

Table 1 shows the energy resolution of the detectors investigated, measured at $511 \mathrm{keV}$ as described in section 2.5.2. Separate studies showed that the energy resolution remains constant within less than $1.5 \%$ FWHM when irradiating the detectors at different positions. The position dependence is indeed expected to be relatively small as the crystals were carefully wrapped in Teflon tape. It is also noted that the energy resolution of crystal R01-P was found to vary by less than $0.3 \%$ FWHM over a $5 \mathrm{~K}$ temperature range. The independently measured absolute light yields of the scintillation crystals (see section 2.5.1) are also listed. These can be seen to be very similar for all crystals studied.

Figure 5 shows an example of a pulse height spectrum, measured at $511 \mathrm{keV}$ with detector R01-P (see section 2.1 for an explanation of the crystal code). The corresponding energy resolution is $10.5 \pm 0.2 \%$ FWHM. The full-energy peak contains $\sim 75 \%$ of the events for all detectors investigated, although the probability of photoelectric interaction of $511 \mathrm{keV}$ photons in LYSO:Ce is only $\sim 30 \%$ of the total probability of interaction. This is due to the large fraction of Compton-scattered photons that are re-absorbed within the relatively large crystal. In a PET system, this has the advantage that the application of an energy threshold to reduce the influence of intra-subject scattering has a relatively small effect on the sensitivity to true events.

A detailed analysis of how different factors such as the scintillation photon statistics, APD dark current, APD excess noise factor, electronic noise, etc, influence the energy resolution of our detectors is presented elsewhere (Maas et al 2008).

\subsection{Timing resolution}

Figure 6 shows a coincidence timing spectrum measured with crystal R01-P against a $\mathrm{BaF}_{2}$-PMT detector (see section 2.5.3). A timing resolution of $2.0 \pm 0.1 \mathrm{~ns}$ FWHM is obtained with this detector. Assuming that the timing resolution of the $\mathrm{BaF}_{2}-\mathrm{PMT}$ detector is negligibly small $(<200 \mathrm{ps})$, the coincidence timing resolution of two of these detectors equals $\Delta t^{\prime}=\sqrt{2} \Delta t=\sim 2.8$ ns FWHM. 


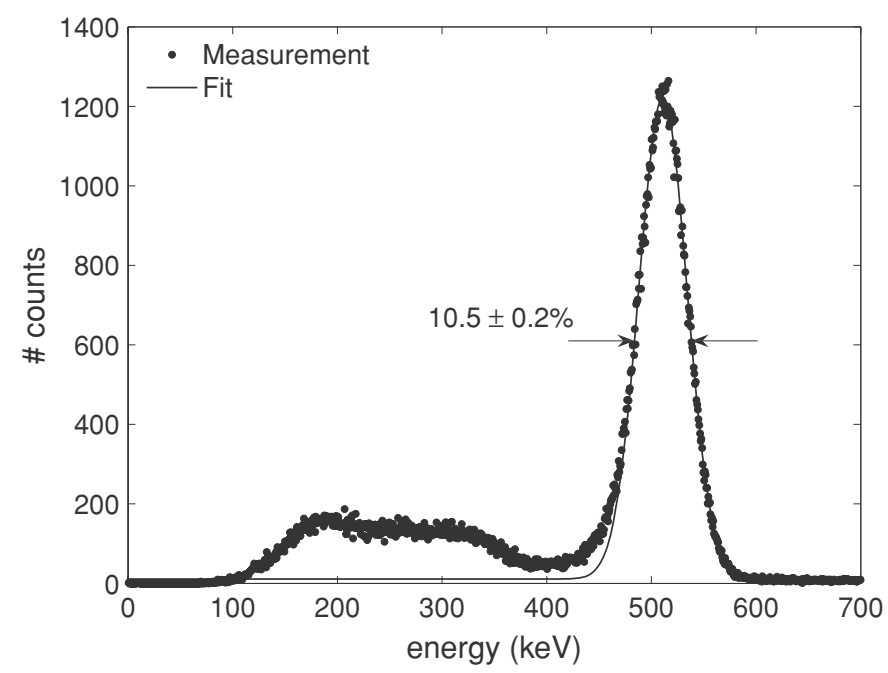

Figure 5. Pulse-height spectrum of detector R01-P measured at $511 \mathrm{keV}$ (dots). A Gaussian fit to the full-energy peak is also shown (solid curve).

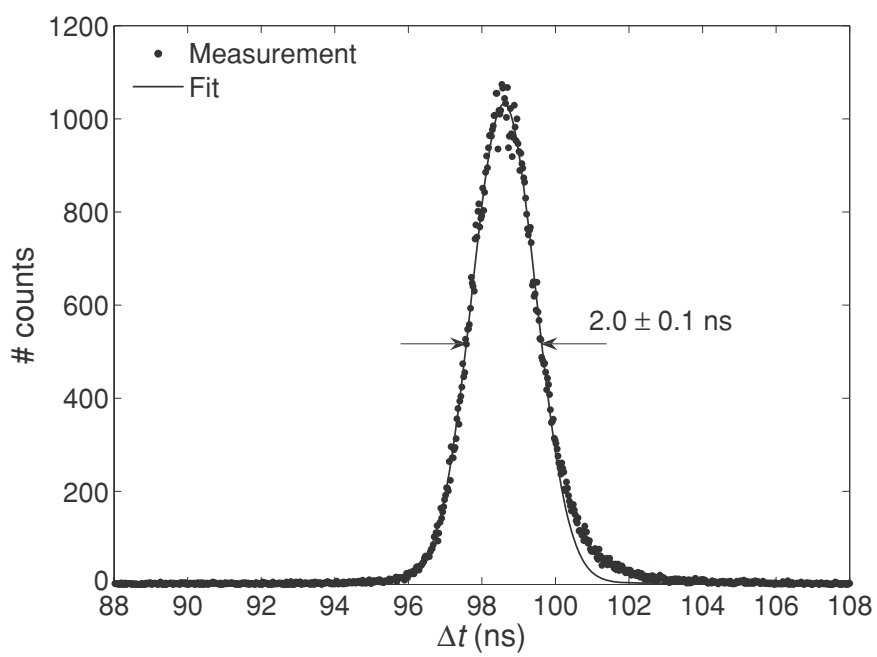

Figure 6. Timing spectrum measured with crystal R01-P against a $\mathrm{BaF}_{2}-\mathrm{PMT}$ detector (dots). A Gaussian fit through the data is also shown (solid curve).

The magnitude of the measured timing resolution is large enough that the influence of optical photon transport within the crystal can be assumed negligible. As previous work (Maas et al 2008) has shown that the major factors determining the timing resolution are the signal amplitude and the electronic noise on the signals, it is expected that relatively small changes to the crystal geometry (e.g. having a different thickness and/or a trapezoidal shape) will not significantly affect the timing resolution.

\subsection{Spatial resolution}

3.3.1. Detector PSF. Figure 7 shows the measured point-spread function $\operatorname{PSF}_{\mathrm{b}}(x, y)$ of crystal R01-P obtained at an energy threshold of $E_{\mathrm{th}} \approx 100 \mathrm{keV}$ and with $L=500$. It will be 


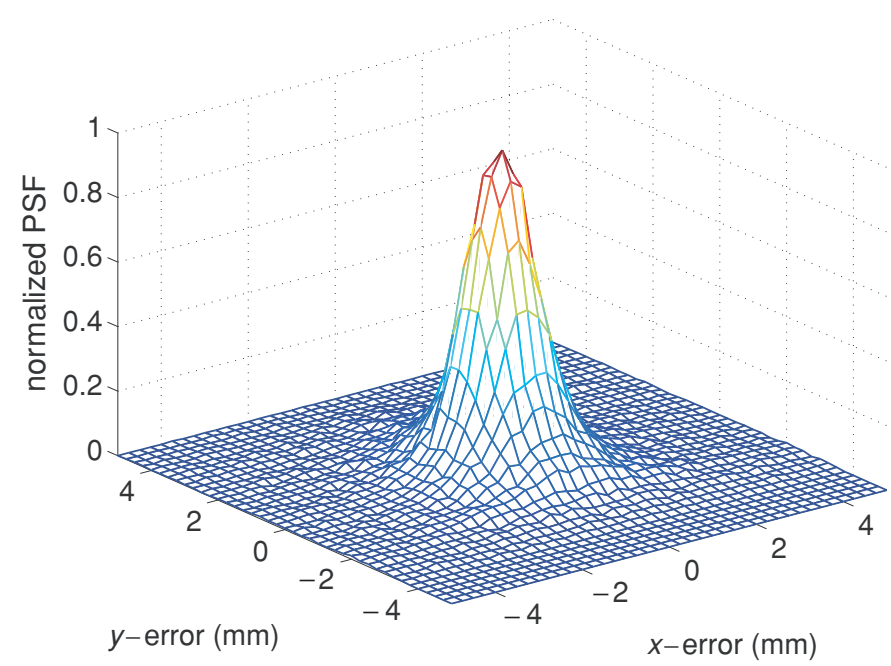

Figure 7. Measured point-spread function $\operatorname{PSF}_{\mathrm{b}}(x, y)$ of crystal R01-P at the detector centre, obtained at normal incidence using $L=500$. The result is not yet corrected for the beam diameter $d_{\mathrm{b}}=0.96 \mathrm{~mm}$ FWHM.

Table 2. FWHM and FWTM of the cross-sections in the $x$ - and $y$-directions of the measured $\left(\mathrm{PSF}_{\mathrm{b}}\right)$ and corrected (PSF) point-spread functions of crystal R01-P at the detector centre and at normal incidence. These results were obtained at room temperature with $L=500$ and $E_{\text {th }} \approx$ $100 \mathrm{keV}$.

\begin{tabular}{llll}
\hline Cross-section & Beam diameter & FWHM (mm) & FWTM (mm) \\
\hline $\operatorname{PSF}_{\mathrm{b}}(x)$ & $0.96 \mathrm{~mm}$ & 1.54 & 3.33 \\
$\operatorname{PSF}_{\mathrm{b}}(y)$ & & 1.61 & 3.80 \\
$\operatorname{PSF}(x)$ & Corrected & 1.05 & 2.09 \\
$\operatorname{PSF}(y)$ & & 1.25 & 2.42 \\
\hline
\end{tabular}

shown below that $\operatorname{PSF}_{\mathrm{b}}(x, y)$ is essentially constant in the central part of the detector: the 2D error histogram shown in figure 7 has therefore been averaged over the region $(-3 \mathrm{~mm}<x<$ $3 \mathrm{~mm}, 0<y<1.5 \mathrm{~mm}$ ) to minimize statistical fluctuations. It is emphasized that the results presented here are not yet corrected for the influence of the beam diameter $d_{\mathrm{b}}=0.96 \mathrm{~mm}$ (see section 2.5.4).

The cross-section of $\operatorname{PSF}_{\mathrm{b}}(x, y)$ in the $x$-direction at zero $y$-error, which will be denoted as $\operatorname{PSF}_{\mathrm{b}}(x)$, has a FWHM of $1.54 \mathrm{~mm}$, see table 2. The perpendicular cross-section $\operatorname{PSF}_{\mathrm{b}}(y)$ has a FWHM of $1.61 \mathrm{~mm}$.

Figure 8 shows the FWHM of $\operatorname{PSF}_{\mathrm{b}}(x)$ as a function of the beam position for $y>0$. As stated before, $\operatorname{PSF}_{\mathrm{b}}(x)$ appears to be essentially constant in the central part of the detector, except for statistical fluctuations arising from the limited number of data points per histogram, and, perhaps, some small variation reflecting the pixel geometry of the APD arrays.

The spatial resolution in the $x$-direction is affected near the two crystal surfaces perpendicular to the $x$-axis. At a distance of about 3-4 $\mathrm{mm}$ from these crystal edges, the FWHM starts to increase, up to an average value of $\sim 1.9 \mathrm{~mm}$ at $\sim 2 \mathrm{~mm}$ from the crystal edges. At still smaller distances, the FWHM decreases again, as the PSF becomes truncated on one side due to the absence of reference data beyond the crystal edges. $\operatorname{PSF}_{\mathrm{b}}(x)$ is only 


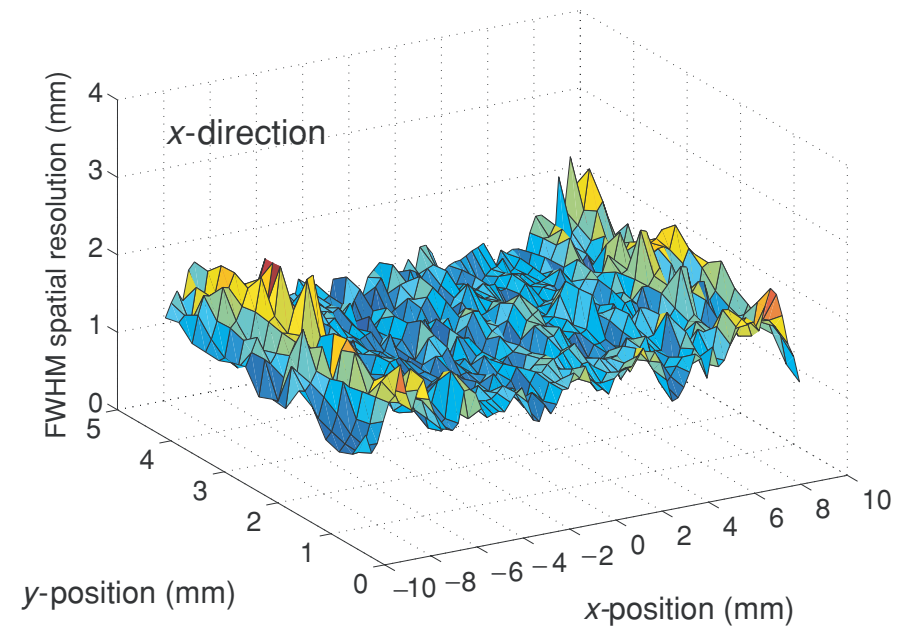

Figure 8. FWHM of $\mathrm{PSF}_{\mathrm{b}}(x)$ of crystal R01-P measured at normal incidence as a function of the test beam position, using $L=500$. Values are derived from 2D error histograms averaged over $1 \times 1 \mathrm{~mm}^{2}$ segments and are not corrected for $d_{\mathrm{b}}=0.96 \mathrm{~mm}$ FWHM.

affected near the crystal edges perpendicular to the $x$-axis. These effects have been predicted by optical simulations (van der Laan et al 2006) and have also been measured with $10 \mathrm{~mm}$ thick crystals read out by a single APD array in FSR or BSR geometry (Maas et al 2006).

Similar results were found in the $y$-direction: the $\operatorname{FWHM}_{\text {of }} \operatorname{PSF}_{\mathrm{b}}(y)$ is best in the central part of the detector, while some deterioration of the spatial resolution in the $y$-direction is found near the crystal edges perpendicular to the $y$-axis only.

3.3.2. Correction for test beam diameter. The results presented in the previous section still include the influence of the finite test beam diameter. This influence can be corrected for as described in section 2.5.5, in order to estimate the detector PSF applicable to individual annihilation photons.

Here we illustrate the procedure for the measured $\mathrm{PSF}_{\mathrm{b}}$ at the centre of detector R01-P shown in figure 7. Figure 9 shows the cross-sections $\operatorname{PSF}_{\mathrm{b}}(x)$ of both the measured $\operatorname{PSF}_{\mathrm{b}}(x, y)$ (closed circles) and the model of $\operatorname{PSF}_{\mathrm{b}}(x, y)$ (dotted curve). Excellent agreement between the model and the measurements is obtained. The cross-section $\operatorname{PSF}(x)$ of the corrected detector $\operatorname{PSF}(x, y)$ is indicated by the solid curve in the same figure.

Table 2 shows the FWHM and FWTM in the $x$ - and $y$-directions of both the uncorrected and the corrected PSFs. A corrected spatial resolution of $1.05 \mathrm{~mm}$ FWHM is found in the $x$-direction. Similar to $\mathrm{PSF}_{\mathrm{b}}$ (see section 3.3.1), the corrected PSF is slightly wider in the $y$-direction.

It is emphasized that these results are obtained at a low energy threshold $E_{\text {th }} \approx 100 \mathrm{keV}$. Separate studies have shown that the influence of $E_{\mathrm{th}}$ on the spatial resolution is very small (i.e. no more than a few hundredths of a mm FWHM). This implies that one can select a low energy threshold for imaging small subjects (with low intra-subject scattering) to maximize the system sensitivity without loss of resolution.

3.3.3. Crystal comparison. The spatial resolutions of the rectangular and trapezoidal crystals were compared in a series of measurements in which the two APD arrays (and other parameters) were kept the same. For efficiency, this was done by means of $1 \mathrm{D}$ resolution measurements 


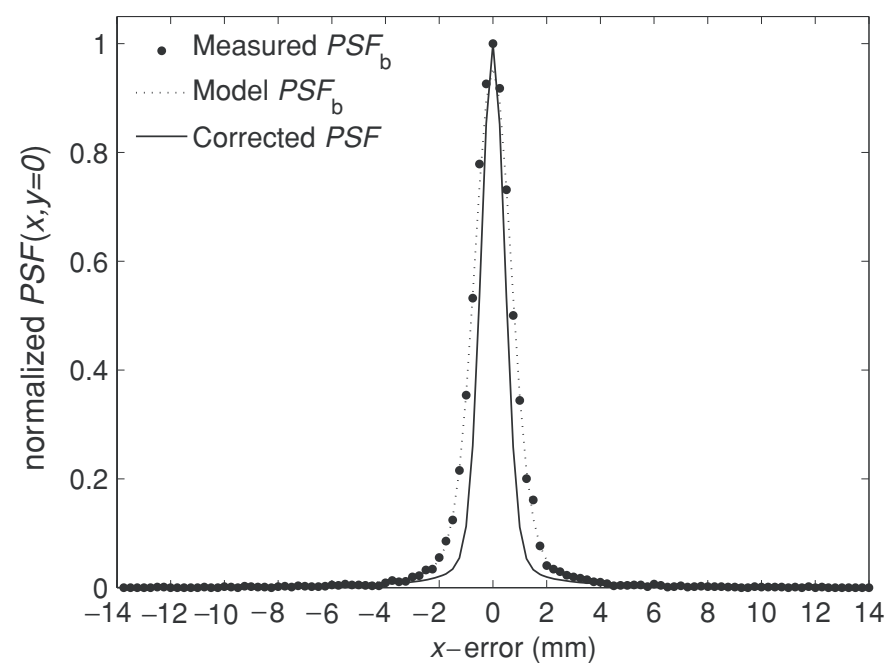

Figure 9. Measured (closed circles) and modelled (dotted curve) uncorrected PSF cross-sections $\operatorname{PSF}_{\mathrm{b}}(x)$, and cross-section $\operatorname{PSF}(x)$ of the corrected PSF (solid curve), derived from the measured $\operatorname{PSF}_{\mathrm{b}}(x, y)$ at the centre of crystal R01-P shown in figure 7.

Table 3. FWHM and FWTM of uncorrected 1D point-spread functions, measured along the $x$ - and $y$-axis of several trapezoidal and rectangular detectors and averaged over the entire crystal length. Results are not corrected for $d_{\mathrm{b}}=0.85 \mathrm{~mm}$ FWHM. The value of $L$ that minimized the FWHM (typically, $100<L<1000$ ) was used in all cases. The estimated uncertainty is $\sim 0.05 \mathrm{~mm}$ for the FWHM and $\sim 0.2 \mathrm{~mm}$ for the FWTM $(2 \sigma)$.

\begin{tabular}{llllll}
\hline & \multicolumn{2}{c}{1 1D-PSF $_{\mathrm{b}}(x)$} & & \multicolumn{2}{c}{1 D-PSF $_{\mathrm{b}}(y)$} \\
\cline { 2 - 3 } \cline { 6 - 6 } Crystal & $\begin{array}{l}\text { FWHM } \\
(\mathrm{mm})\end{array}$ & $\begin{array}{l}\text { FWTM } \\
(\mathrm{mm})\end{array}$ & & $\begin{array}{l}\text { FWHM } \\
(\mathrm{mm})\end{array}$ & $\begin{array}{l}\text { FWTM } \\
(\mathrm{mm})\end{array}$ \\
\hline R01-P & 1.78 & 5.07 & & 1.93 & 5.68 \\
T02-P & 1.84 & 5.15 & & 1.88 & 6.17 \\
T03-A & 1.86 & 5.73 & & 1.92 & 7.09 \\
\hline
\end{tabular}

as explained in section 2.5.4. It is emphasized that the $1 \mathrm{D}$ resolutions presented in this section cannot be compared directly to the $2 \mathrm{D}$ results at the centre of the detector given in section 3.3.1. This is both because of the non-Gaussian shape of the PSFs and because the $1 \mathrm{D}$ results presented in this section are averaged over the entire crystal length, as discussed below. As a consequence, the 1D results tend to be at least several tenths of a mm FWHM larger in otherwise equal measurements. They are nevertheless useful to study the variation of the spatial resolution with the parameter of interest (i.e. the crystal type).

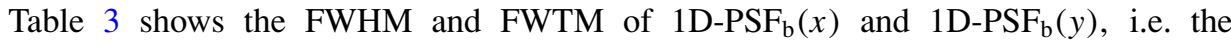
uncorrected 1D PSFs measured along the $x$ - and the $y$-axis, respectively, for all crystals investigated. The error histograms were averaged over the entire length of the crystal, so that they represent the overall detector performance. As we are only interested in the variation of the spatial resolution between different crystals, no correction for the beam diameter was applied to avoid introducing additional uncertainties. A fair comparison between the different detectors was assured by keeping $d_{\mathrm{b}}=0.85 \mathrm{~mm}$ FWHM the same in all measurements. As in section 3.3.1, average resolutions in the $y$-direction appear to be slightly worse than in the $x$-direction. 


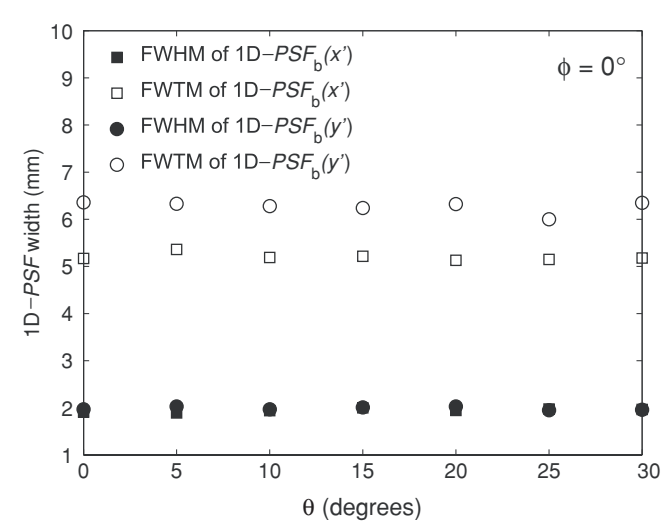

(a)

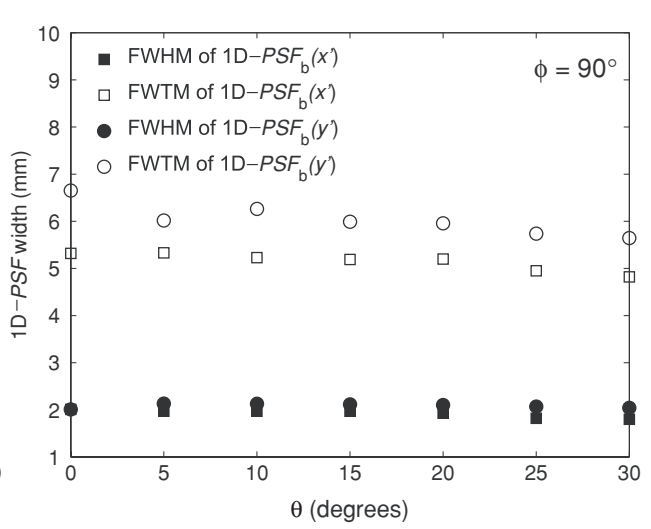

(b)

Figure 10. FWHM and FWTM of $1 \mathrm{D}-\mathrm{PSF}_{\mathrm{b}}\left(x^{\prime}\right)$ and $1 \mathrm{D}-\mathrm{PSF}_{\mathrm{b}}\left(y^{\prime}\right)$ of crystal T02-P, averaged over the entire crystal length and projected onto a plane perpendicular to the test beam, as a function of the angle of incidence $\theta$ at (a) $\varphi=0^{\circ}$ and (b) $\varphi=90^{\circ}$. Results are not corrected for $d_{\mathrm{b}}=$ $0.96 \mathrm{~mm}$ FWHM. The estimated uncertainty is $\sim 0.05 \mathrm{~mm}$ for the FWHM and $\sim 0.2 \mathrm{~mm}$ for the FWTM $(2 \sigma)$

Comparing the two trapezoidal crystals with different surface finishes, we observe that the FWHM values are the same within the measurement uncertainty $(2 \sigma \approx 0.05 \mathrm{~mm})$. However, the FWTM values of the as-cut trapezoidal crystal are significantly larger than those of the polished one $(2 \sigma \approx 0.2 \mathrm{~mm})$.

Comparing the polished trapezoidal crystal with the polished rectangular crystal, the differences appear to be comparable to the measurement uncertainties except for FWTM in the $y$-direction, which is slightly but significantly $(2 \sigma \approx 0.2 \mathrm{~mm})$ larger for the trapezoidal crystal. This is attributed to events occurring near the oblique sides of the trapezoidal crystal.

It is concluded that polished trapezoidal crystals perform almost as well as rectangular crystals, while minimizing the dead space within a detector ring (van der Laan et al 2007).

3.3.4. Depth of interaction correction. So far, we have presented the detector spatial resolution in terms of the uncertainties in the coordinates $x$ and $y$ defined within the plane of the crystal front surface (see figure 1). At normal incidence, these coincide with the uncertainties in the position of the line of response (LOR) in a PET system. To study the spatial resolution as a function of the angle of incidence $\theta$, however, the uncertainties must be projected onto a plane perpendicular to the LOR (or the beam axis in our test setup). We therefore define coordinates $x^{\prime}$ and $y^{\prime}$ within this plane such that $x^{\prime}=x \cos (\theta)$ and $y^{\prime}=y$ at $\varphi=0^{\circ}$, while $x^{\prime}=x$ and $y^{\prime}=y \cos (\theta)$ at $\varphi=90^{\circ}$.

The DOI correction of our detectors was studied in a series of 1D measurements. Figure 10 shows the FWHM and FWTM of $1 \mathrm{D}-\mathrm{PSF}_{\mathrm{b}}\left(x^{\prime}\right)$ and $1 \mathrm{D}-\mathrm{PSF}_{\mathrm{b}}\left(y^{\prime}\right)$ of the trapezoidal crystal T02-P as a function of $\theta$. As in the previous section, the error histograms were averaged over the crystal length to represent the overall detector performance. The values are not corrected for the beam diameter, but care was taken to keep $d_{\mathrm{b}}$ constant in all measurements. The source had to be moved some distance away from the detector box to accommodate for its rotation, so $d_{\mathrm{b}}=0.96 \mathrm{~mm}$ FWHM and, therefore, these results cannot be compared directly to those in table 3 .

At $\varphi=0^{\circ}$, both $1 \mathrm{D}-\mathrm{PSF}_{\mathrm{b}}\left(x^{\prime}\right)$ and $1 \mathrm{D}-\operatorname{PSF}_{\mathrm{b}}\left(y^{\prime}\right)$ are essentially independent of the angle of incidence. At $\varphi=90^{\circ}, 1 \mathrm{D}-\mathrm{PSF}_{\mathrm{b}}\left(x^{\prime}\right)$ can even be seen to slightly improve with increasing 
$\theta$. This is attributed to the fact that in these experiments the beam exits the side surface of the crystal at large values of $\theta$, reducing the average distance of interaction from the front APD array. Similar results were found for the rectangular crystals, although the difference between the FWTMs of $1 \mathrm{D}-\mathrm{PSF}_{\mathrm{b}}\left(x^{\prime}\right)$ and $1 \mathrm{D}-\mathrm{PSF}_{\mathrm{b}}\left(y^{\prime}\right)$ was smaller for these crystals, in accordance with the results given in the previous section.

It is concluded that the excellent intrinsic DOI correction of our detectors enables essentially parallax-free image reconstruction at angles of incidence of up to at least $30^{\circ}$.

\section{Conclusions}

Monolithic scintillator PET detectors with intrinsic DOI correction were characterized experimentally in terms of their spatial, temporal and energy resolutions. The detector design comprises a rectangular or trapezoidal LYSO:Ce crystal read out by two position-sensitive APD arrays in double-sided readout geometry. This involves placement of one of the sensors on the crystal front surface, which is feasible because the APD arrays are essentially transparent to $511 \mathrm{keV}$ photons. With DSR the crystal can be made $20 \mathrm{~mm}$ thick for maximum detection efficiency, while maintaining high spatial resolution.

At the centre of the detector and at normal incidence, the FWHM of the detector PSF obtained with a rectangular crystal is as good as $\sim 1.05 \mathrm{~mm}$ in the $x$-direction, after correction for the $\sim 0.9 \mathrm{~mm}$ diameter test beam of annihilation photons. Near the edges of the crystal some resolution loss occurs, in the direction perpendicular to the edge only.

Trapezoidal crystals perform almost equally well as rectangular ones. Such crystals minimize the dead space within a detector ring, resulting in the highest and most uniform scanner sensitivity (van der Laan et al 2007).

No loss of spatial resolution is observed for angles of incidence of up to at least $30^{\circ}$. The excellent DOI correction thus demonstrated will facilitate a high and uniform PET system resolution.

These promising results were obtained at $E_{\mathrm{th}} \approx 100 \mathrm{keV}$, implying that one can use a low energy threshold for imaging small subjects (with low intra-subject scattering), so as to maximize system sensitivity without loss of resolution.

Energy resolutions of $\sim 11 \%$ FWHM are measured with $\sim 75 \%$ of the events in the fullenergy peak. The estimated coincidence timing resolution of $\sim 2.8 \mathrm{~ns}$ FWHM is sufficient for adequate rejection of random coincidences (van der Laan et al 2007).

Whereas some PET detector concepts might offer even higher spatial resolution at the centre of the FOV, our monolithic detectors offer the advantages of highly accurate DOI correction, high detection efficiency, good energy resolution and simplicity of design. Because of these advantages they are expected to outperform many other detectors, especially in applications where larger, or multiple, objects need to be imaged with high and uniform resolution. As an added advantage, the use of APD arrays instead of (multi-channel) PMTs opens up the possibility of integrating these detectors with an MRI device.

\section{References}

Abreu M et al 2006 Design and evaluation of the Clear-PEM scanner for positron emission mammography IEEE Trans. Nucl. Sci. 53 71-7

Bloomfield P M, Myers R, Hume S P, Spinks T J, Lammertsma A A and Jones T 1997 Three-dimensional performance of a small-diameter positron emission tomograph Phys. Med. Biol. 42 389-400

Bruyndonckx P, Léonard S M A, Liu J, Tavernier S P K, Szupryczynski P and Fedorov A 2003 Study of spatial resolution and depth of interaction of APD-based PET detector modules using light sharing schemes IEEE Trans. Nucl. Sci. $\mathbf{5 0} 1415-9$ 
Bruyndonckx P, Léonard S M A, Tavernier S P K, Lemaître C, Devroede O, Wu Y and Krieguer M 2004 Neural network-based position estimators for PET detectors using monolithic LSO blocks IEEE Trans. Nucl. Sci. $512520-5$

Catana C, Wu Y, Judenhofer M S, Qi J, Pichler B J and Cherry S R 2006 Simultaneous acquisition of multislice PET and MR images: initial results with a MR-compatible PET scanner J. Nucl. Med. 47 1968-76

Clément D, Frei R, Loude J and Morel C 1998 Development of a 3D position sensitive scintillation detector using neural networks 1998 IEEE Nucl. Sci. Symp. Conf. Record pp 1448-52

Correia J A, Burnham C A, Kaufman D and Fischman A J 1999 Development of a small animal PET imaging device with resolution approaching $1 \mathrm{~mm}$ IEEE Trans. Nucl. Sci. 46 631-5

Cover T M and Hart P E 1967 Nearest neighbor pattern classification IEEE Trans. Inf. Theory 13 21-7

De Haas J, Dorenbos P and Van Eijk C W E 2005 Measuring the absolute light yield of scintillators Nucl. Instrum. Methods Phys. Res. A 537 97-100

Del Guerra A, Di Domenico G, Scandola M and Zavatti G 1998 High spatial resolution small animal YAP-PET Nucl. Instrum. Methods Phys. Res. A $409537-41$

Du H, Yang Y and Cherry S R 2008 Comparison of four depth-encoding PET detector modules with wavelength shifting (WLS) and optical fiber read-out Phys. Med. Biol. 53 1829-42

Judenhofer M S et al 2008 Simultaneous PET-MRI: a new approach for functional and morphological imaging Nat. Med. 14 459-65

Lecomte R, Cadorette J, Rodrique S, Lapointe D, Rouleau D, Bentourkia M, Yao R and Msaki P 1996 Initial results from the Sherbrooke avalanche photodiode positron tomograph IEEE Trans. Nucl. Sci. 43 1952-7

Maas M C 2008 Monolithic scintillator detectors for high-resolution positron emission tomography PhD Thesis Delft University of Technology, Delft, The Netherlands

Maas M C, Schaart D R, Van Der Laan D J, Van Dam H T, Huizenga J, Brouwer J C, Bruyndonckx P, Lemaître C and Van Eijk C W E 2008 Signal to noise ratio of APD-based monolithic scintillator detectors for high resolution PET IEEE Trans. Nucl. Sci. 55 842-52

Maas M C, Van Der Laan D J, Schaart D R, Huizenga J, Brouwer J C, Bruyndonckx P, Léonard S, Lemaître C and Van Eijk C W E 2006 Experimental characterization of monolithic-crystal small animal PET detectors read out by APD arrays IEEE Trans. Nucl. Sci. 53 1071-7

McElroy D P, Pimpl W, Pichler B J, Rafecas M, Schuler T and Ziegler S I 2005 Characterization and readout of MADPET-II detector modules: validation of a unique design concept for high resolution small animal PET IEEE Trans. Nucl. Sci. 52 199-204

Orita N, Murayama H, Kawai H, Inadama N and Tsuda T 2005 Three-dimensional array of scintillation crystals with proper reflector arrangement for a depth of interaction detector IEEE Trans. Nucl. Sci. 52 8-14

Seidel J, Vaquero J J and Green M V 2003 Resolution uniformity and sensitivity of the NIH ATLAS small animal PET scanner: comparison to simulated LSO scanners without depth-of-interaction capability IEEE Trans. Nucl. Sci. 50 1347-51

Surti S, Karp J S, Perkins A E, Freifelder R and Mühllehner G 2003 Design evaluation of A-PET: a high sensitivity animal PET camera IEEE Trans. Nucl. Sci. 50 1357-63

Tai Y C, Chatziioannou A F, Yang Y, Silverman R W, Meadors K, Siegel S, Newport D F, Stickel J R and Cherry S R 2003 MicroPET II: design, development and initial performance of an improved MicroPET scanner for small-animal imaging Phys. Med. Biol. 49 1519-39

Tai Y C, Ruangma A, Rowland D, Siegel S, Newport D F, Chow P L and Laforest R 2005 Performance evaluation of the MicroPET focus: a third generation MicroPET scanner dedicated to animal imaging J. Nucl. Med. 46 455-63

Van Der Laan D J, Maas M C, De Jong H W A M, Schaart D R, Bruyndonckx P, Lemaître C and Van Eijk C W E 2007 Simulated performance of a small-animal PET scanner based on monolithic scintillation detectors $\mathrm{Nucl}$. Instrum. Methods Phys. Res. A $571227-30$

Van Der Laan D J, Maas M C, Schaart D R, Bruyndonckx P, Léonard S and Van Eijk C W E 2006 Using Cramér-Rao theory combined with Monte Carlo simulations for the optimization of monolithic scintillator PET detectors IEEE Trans. Nucl. Sci. 53 1063-70

Wang G C, Huber J, Moses W, Choong W S and Maltz J 2004 Calibration of a PEM detector with depth of interaction measurement IEEE Trans. Nucl. Sci. 51 775-81

Weber S, Bauer A, Herzog H, Kehren F, Mühlensiepen H, Vogelbruch J, Coenen H H, Zilles K and Halling H 1999 Recent results of the TierPET scanner 1999 IEEE Nucl. Sci. Symp. Conf. Record pp 1603-7

Woody C et al 2007 Preliminary studies of a simultaneous PET/MRI scanner based on the RatCAP small animal tomograph Nucl. Instrum. Methods Phys. Res. A 571 102-5

Ziemons K et al 2005 The ClearPET (TM) project: development of a 2nd generation high-performance small animal PET scanner Nucl. Instrum. Methods Phys. Res. A 537 307-11 